\title{
De la désaffiliation du chercheur pour l'élaboration des connaissances : comment se positionner en tant que chercheur et acteur dans une école ethno-religieuse? \\ Researcher's disaffiliation for the development of knowledge: How to position yourself as a researcher and actor in an ethno-religious school?
}

\author{
Souheil Essid et Yosra Essid Hamas
}

Volume 13, numéro 1, novembre 2017

Sur le thème de la recherche sur la recherche

URI : https://id.erudit.org/iderudit/1044013ar

DOI : https://doi.org/10.7202/1044013ar

\section{Aller au sommaire du numéro}

\section{Éditeur(s)}

Prise de parole

\section{ISSN}

1712-8307 (imprimé)

1918-7475 (numérique)

\section{Découvrir la revue}

Citer cet article

Essid, S. \& Essid Hamas, Y. (2017). De la désaffiliation du chercheur pour l'élaboration des connaissances : comment se positionner en tant que chercheur et acteur dans une école ethno-religieuse? Nouvelles perspectives en sciences sociales, 13(1), 117-148. https://doi.org/10.7202/1044013ar

\section{Résumé de l'article}

Quand le chercheur s'investit dans son action de recherche, il entretient un rapport cognitif et affectif avec son objet d'étude. Dans cet article, nous souhaitons aborder comment la question de l'implication du chercheur est un enjeu de la recherche et en quoi l'analyse des processus à l'oeuvre pour le chercheur peut devenir un objet de réflexion qui permet aussi une compréhension de l'objet d'étude. Dans le cadre d'une recherche doctorale sur le rôle de la religion et de l'école dans la construction identitaire des jeunes, notre immersion dans une école primaire au Québec nous place au coeur d'un processus réflexif sur la posture du chercheur, sur sa réflexivité et sur son positionnement par rapport à soi-même, à ses sujets de recherche et à ses outils de collecte de données. Dans cette étude, l'observation ethnographique comme outil de collecte de données nous a placé au coeur d'un processus conversationnel continu avec les sujets, avec les données et avec soi-même. Le processus a conduit le chercheur à une désaffiliation religieuse et à une réflexion sur les aspects intersubjectifs de la recherche. 


\section{De la désaffiliation du chercheur pour l'élaboration des connaissances : comment se positionner en tant que chercheur et acteur dans une école ethno-religieuse?}

SOUHEIL ESSID

College of education, Taibah University, KSA

YosRa EsSid Hamas

College of business, Effat University, KSA

"Le chercheur ne chercherait que lui-même. Il est son premier objet de recherche $[. .$.$] il est aussi destiné au sacrifice et, s'il veut$ reconnaître l'autre, il lui faudra d'abord passer par lui-même "

Jacqueline Barus-Michel ${ }^{1}$

D ans une recherche doctorale portant sur l'expérience identitaire de jeunes qui fréquentent une école ethno-religieuse ${ }^{2}$ dans la province du Québec, Souhaiel Essid a voulu mieux comprendre le processus de la construction de l'identité religieuse de jeunes Canadiens issus de l'immigration musulmane. L'objectif de départ consistait à comprendre la réalité scolaire et sociale des jeunes à partir de leurs expériences en milieu religieusement minoritaire, à savoir une école musulmane dans une société

1 Jacqueline Barus-Michel, "Le chercheur, premier objet de la recherche ", Bulletin de psychologie, vol. 39, n 377, 1986, p .801.

2 Une école privée qui livre le programme officiel du Québec, mais fréquentée essentiellement par une clientèle religieuse et/ou ethnique. 
occidentale ${ }^{3}$. La fréquentation d'une école musulmane à temps plein constitue un élément essentiel du processus de construction de cette identité qui se situe dans une tension entre l'appartenance à une communauté, minorisée par ses origines et ses croyances religieuses, et l'intégration à la société plus large dans laquelle elle s'inscrit.

Étant lui-même membre de la communauté musulmane, il a espéré que le choix de cette communauté comme terrain de recherche faciliterait son travail comme chercheur. Ainsi, il s'est considéré mieux placé pour faire une étude sur cette communauté en raison des points communs qu'ils partagent, notamment la religion et l'expérience migratoire. Lesley Milroy évoque l'avantage des chercheurs qui entreprennent des recherches sur leurs communautés, linguistique dans son cas, et insiste sur l'importance de l'enquête linguistique pour comprendre les normes et

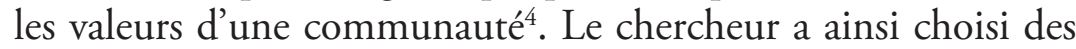
participants avec qui il partage la même religion et en partie l'expérience migratoire. Ce partage du répertoire ne nécessite pas l'intervention d'autres acteurs, puisque les chercheurs comme membres de groupe connaissent bien la culture du groupe en question en tant que membres et en tant qu'acteurs actifs 5 .

Mais, une fois sur le terrain d'enquête, il a pris conscience que l'avantage du chercheur ne réside nécessairement pas dans son appartenance au groupe de recherche, mais dans sa démarche d'objectivation, de réflexivité et de distanciation par rapport à son objet de recherche et par rapport à sa propre démarche épistémologique et méthodologique. Jean Foucart l'a déjà confirmé : "La construction de la recherche sociale ne peut se faire ni entièrement du côté de l'immersion, ni du côté de la distanciation. Elle est dans la démarche de l'entre-deux ${ }^{6} »$. En

3 Souhaiel Essid, La construction de l'identité dans une école indépendante. Le cas d'une école musulmane à Montréal, thèse de doctorat, Ottawa, Université d'Ottawa, 2015.

4 Lesley Milroy, Language and Social Networks, Oxford, Blackwell, 1980, p. 17. Ibid.

Jean Foucart, "La recherche sociale entre immersion et distanciation ", Pensée plurielle, vol. 2, nos 30-31, 2012, p. 280. 
effet, tout au long de son étude, le chercheur s'est trouvé au cœur d'un processus conversationnel continu tant avec les sujets, qu'avec les données, mais aussi dans un dialogue interne avec lui-même. Ce concept de " processus conversationnel » est emprunté à l'approche interprétative, particulièrement narrative de Barbara Czarniawska qui décrit la recherche et les sciences sociales en général comme une conversation ${ }^{7}$. Ainsi, à travers la conversation, le chercheur étudie la façon dont les acteurs construisent leur monde : les faits, les explications, ou peut-être les histoires et les interprétations. Une fois que l'idée de la structure de la construction est plus claire, il contribue à la conversation par la publication et la joint en recevant des commentaires, des critiques et des réactions ${ }^{8}$.

Grâce à ce processus conversationnel, des rapports dynamiques à la fois d'immersion et de distanciation ont été vécus durant l'étude. Ainsi, les choix de départ (sujet et question de recherche, méthodologie et design de la recherche, etc.) reflètent des choix, principalement subjectifs, du chercheur, de sa personnalité, de son histoire et de ses connaissances théoriques et expériences pratiques. Une fois sur terrain, ces choix se confrontent aux réalités sociales et relationnelles et certains se trouvent parfois déroutés. Dans ce cadre, la réflexivité du chercheur lui permet d'apporter une nouvelle dimension à la recherche, à savoir d'entretenir avec les données et avec les sujets des rapports dynamiques à la fois de distanciation et de rapprochement. Cette conversation lui permet d'observer son influence sur les sujets et la redécouverte des similitudes et des différences avec les acteurs. $\mathrm{Au}$ cours de cette recherche, le chercheur a pris conscience que l'appartenance à la même culture constitue un stéréotype, puisqu'il n'y a pas une culture musulmane unique. Le processus d'objectivation des données ressemblerait davantage à un processus de subjectivation permettant la redécouverte de l'objet de recherche lui-même en donnant sens aux données recueillies. D’où résulte

\footnotetext{
$7 \quad$ Barbara Czarniawska, Narrating the Organization: Dramas of Institutional Identity, Chicago, The University of Chicago Press, 1997. 
un principe fondamental pour le chercheur : le processus de découverte de soi-même et la remise en cause de ses a priori, de ses propres idées et valeurs, de sa religion et de sa recherche, en général. L'interaction avec les acteurs de la recherche lui a permis de prendre davantage conscience de ses propres valeurs qui se voient parfois nuancées, voire défiées par les observations, les propos ou les actions des participants.

Ces rapports dynamiques de rapprochement et de distanciation, caractéristiques des différents moments de la recherche, leurs manifestations et leurs implications en termes de réflexivité et d'intersubjectivité feront l'objet des sections qui suivent. Nous commençons alors par analyser les fondements théoriques ainsi que la posture méthodologique adoptés par le chercheur pour pouvoir comprendre les prémisses du processus conversationnel. Ensuite, nous nous interrogeons sur le statut du chercheur par rapport à son objet de recherche et à son positionnement face à soi-même. Enfin, nous expliquons l'intersubjectivité du chercheur et sa démarche de réflexivité à travers sa vigilance ethnographique afin de vérifier l'impact de cette démarche sur ses résultats de recherche.

\section{Fondements théoriques et méthodologiques : entre distanciation et immersion}

Partant de l'objectif de vouloir comprendre comment les acteurs construisent leur identité musulmane dans une école confessionnelle particulière et comment ils voient le rôle de leur école dans ce processus, plusieurs choix méthodologiques ont été faits a priori afin de préparer l'entrée au terrain et l'investigation de l'objet de recherche. Les principaux choix méthodologiques du chercheur se résument en fait en une approche qualitative interprétative matérialisée par la méthode de l'étude de cas. La collecte de données a été enrichie par des outils empruntés à l'ethnographie. 


\subsection{Fondements épistémologiques de la recherche}

Pour qu'un objet de recherche aboutisse, des questions sur les fondements théoriques et sur le positionnement du chercheur par rapport à son objet de recherche s'imposent. Vu la diversité des méthodes de recherche et la complexité des sujets, le choix d'une méthode appropriée demande une réflexion profonde sur la démarche de sélection de la méthode, qui doit prendre en compte les différents facteurs qui l'influencent.

Face à un problème de recherche, la question qui se pose souvent est relative au type de recherche qui convient le mieux au chercheur et à l'objet de sa recherche. Le type de recherche de même que la méthode de recherche dépendent ainsi souvent du statut philosophique, épistémologique et méthodologique du chercheur lui-même. Le travail du chercheur consiste donc à adopter une approche qui lui permet d'atteindre ses buts. Son positionnement et les fondements sur lesquels il prend appui constituent le fond du débat philosophique des chercheurs. Il est donc essentiel de s'interroger sur la posture du chercheur par rapport aux paradigmes majeurs qui peuvent englober sa vision du monde?.

Le chercheur, dans notre cas est parti d'une approche constructiviste de la réalité et des connaissances scientifiques selon laquelle le monde social est " fait d'interprétations, construites grâce aux interactions entre acteurs, dans des contextes toujours particuliers ${ }^{10} »$. Pour créer la connaissance, il faut d'abord comprendre le sens que les sujets de la recherche donnent à la réalité et, ensuite, mettre davantage l'accent sur la "construction" de la réalité. C'est justement ce que le chercheur a tenté de réaliser en tentant de comprendre comment les jeunes construisent leurs

9 Pierre Bourdieu, "The Practice of Reflexive Sociology ", dans Pierre Bourdieu and Löic J. D. Wacquant, An Invitation to Reflexive Sociology, Cambridge, Polity, 1992, p. 217-260.

10 Martine Girod-Séville et Véronique Perret, "Fondements épistémologiques de la recherche ", Raymond-Alain Thiétart (dir.), Méthodes de recherche en management, Paris, Dunod, 1999, p. 19. 
identités et le sens qu'ils donnent à ce qu'ils reçoivent dans leur processus de socialisation à cette identité et à ce qui la construit.

Afin d'assurer la vérité des connaissances ${ }^{11}$, une méthode de recherche rigoureuse s'impose; d'où le choix d'une approche qualitative interprétative. Dans cette approche, tout peut a priori être significatif, permettant une ouverture vers des dimensions non repérées d'avance, mais non moins présentes. En saisissant la réalité telle que vécue par les sujets de la recherche, cette démarche favorise une compréhension interne et profonde de la réalité. De plus, la tradition de recherche qualitative est vaste et présente de multiples possibilités sur le plan de la méthodologie. L'ensemble de ces méthodes se rejoint cependant autour d'un objectif commun : " arriver à une perception approfondie des réalités humaines et sociales et en dégager une signification qui correspond à la perspective que les acteurs sociaux ont euxmêmes de ces réalités ${ }^{12}$ ". L'objectif et le positionnement du chercheur se manifestent dans les implications épistémologiques de la recherche et des méthodes employées pour la mettre en œuvre.

Dans les paragraphes qui suivent, nous mettons l'accent sur la méthode adoptée pour expliquer le phénomène de la construction identitaire dans les écoles ethno-religieuses, en termes de type de données, de source des données et de moyens d'analyse.

\subsection{Méthode de recherche : une étude de cas}

Répertoriées au premier rang des méthodes qualitatives, les études de cas sont des études empiriques centrées sur des cas définis en tant que systèmes délimités ${ }^{13}$. En sciences humaines et sociales, le terme "étude de cas ", traduit de l'anglo-saxon, renvoie à une méthode d'investigation qui " consiste à étudier en

\footnotetext{
11 Edmond Marc, "Connaissance de la vérité et vérité de la connaissance ", Société française de Gestalt, vol. 1, nº 34, 2008, p. 11-28.

12 Yves Poisson, La recherche qualitative en éducation, Québec, Presses de l'Université du Québec, 1991, p. 58.

13 Robert Stake, «Case Studies», dans Norman Denzin et Yvonna Lincoln (dir.), Handbook of Qualitative Research, Thousand Oaks (CA), SAGE, 2000, p. 435-454.
} 
détail l'ensemble des caractéristiques d'un phénomène restreint et précis tel qu'il se soit déroulé dans une situation particulière, réelle ou reconstituée, jugée représentative de l'objet à étudier ${ }^{14}$ ". Selon Jacques Hamel, l'ancrage de l'étude de cas dans les sciences humaines et sociales la rattache aux courants de recherche à visée d'analyse et de compréhension, plutôt que de démonstration ou d'explication ${ }^{15}$. En s'appuyant sur des démarches à dominante interprétative, l'étude de cas peut être considérée comme une approche susceptible d'englober plusieurs méthodes et techniques de recueil de données (observation directe, analyse de contenu, enquête par questionnaires et entretiens), sans pour autant comporter un protocole méthodologique strict, comme c'est le cas dans l'approche expérimentale. Son aspect empirique conduit à focaliser la collecte de données sur le vécu et le quotidien des acteurs afin de comprendre une situation en profondeur ${ }^{16}$. Dans le même sens, Jacques Hamel explique que "l'étude de cas consiste à rapporter un phénomène à son contexte et à l'analyser pour voir comment il s'y manifeste et se développe ${ }^{17}$ ». Quant à Martyn Hammersley et Paul Atkinson, ils donnent un caractère ethnographique à l'étude de cas vu le poids attribué au sens que les acteurs donnent eux-mêmes à leur conduite plutôt qu’à l'analyse externe du chercheur ${ }^{18}$. Selon Martyn Hammersley et Roger, la démarche du chercheur consisterait plus à « donner une voix aux acteurs qu’à les utiliser comme informateurs ou des répondants ${ }^{19} \%$.

$\overline{14}$ Brigitte Albero, "L'étude de cas : une modalité d'enquête difficile à cerner ", dans Brigitte Albero et Nicole Poteaux (dir.), Enjeux et dilemmes de l'autonomie. Une expérience d'autoformation à l'université : étude de cas, Paris, Maison des Sciences de l'Homme, coll. "praTICs ", 2010, p. 15.

15 Jacques Hamel, Étude de cas et sciences sociales, Paris, L'Harmattan, coll. " Outils de recherche ", 1997.

16 Yves Poisson, op. cit.

17 Jacques Hamel, op. cit., p. 123.

18 Martyn Hammersley et Paul Atkinson, Ethnography: Principles in Practice, $3^{\mathrm{e}}$ édition, Londres, Routledge, 2007.

19 Martyn Hammersley et Roger Gomm, "Introduction ", dans Roger Gomm, Martyn Hammersley et Peter Foster (dir.), Case Study Method: Key Issues, Key Texts, Londres, SAGE, 2000, p. 3. 


\subsection{La saveur ethnographique}

Pour être fidèle à son souci d'immersion dans les données du terrain, le chercheur a choisi d'approfondir son étude de cas par le recours à des moyens de cueillette de données à saveur ethnographique. Il a ainsi utilisé, entre autres, l'observation, les notes de terrains et les discussions de groupes pour la collecte des données.

Pour distinguer l'étude de cas de l'ethnographie, Sue Walters note que n'est pas toujours clair ce qui différencie les termes " ethnographie " et " étude de cas " ${ }^{20}$. Pour John Creswell, l'ethnographie est la description et l'interprétation d'un groupe ou d'un système culturel ou social dans lequel le chercheur étudie les significations du comportement, du langage et des interactions du groupe dans un contexte particulier ${ }^{21}$. Sue Walters considère que les études ethnographiques sont plus vastes dans la méthode qualitative, alors que l'étude de cas est une stratégie de sélection dans le cadre de l'enquête ${ }^{22}$. L'auteur explique plus loin que l'ethnographie offre une vision holistique d'un groupe social, mais que l'étude de cas offre l'approfondissement d'un système délimité ou d'un ensemble de $\operatorname{cas}^{23}$. Cette différenciation entre les deux approches a permis au chercheur de clarifier son positionnement à l'égard de sa recherche, qui se trouve entre la volonté de détachement et d'objectivation (étude de cas) et la volonté d'immersion dans le terrain (ethnographie).

La recherche présentée représente alors une étude de cas dans la mesure où elle vise des élèves issus de l'immigration musulmane ainsi que leur processus de construction identitaire dans une école dite "musulmane » au Canada. Cependant, elle ne se limite pas aux constructions observées des participants, mais elle

20 Sue Walters, "Case Study" or "Ethnography"? Defining Terms, Making Choices and Defending the Worth of a Case ", dans Geoffrey Walford (dir.), Methodological Developments in Ethnography (Studies in Educational Ethnography), vol. 12, Emerald Group Publishing Limited, 2007, p. 89.

21 John Creswell, Qualitative Inquiry and Research Design: Choosing among Five Traditions, Thousand Oaks (CA), SAGE, 1998.

22 Sue Walters, op. cit., p. 89.

23 Ibid., p. 92. 
cherche à donner voix à ces acteurs et à leurs interprétations. Elle concerne aussi un contexte plus vaste, celui du positionnement de l'Islam en Occident, du rôle des écoles musulmanes dans la transmission et la reproduction de l'Islam, ainsi que le rapport qu'entretiennent les jeunes musulmans avec leurs pays adoptifs et avec l'Occident en général.

La démarche ethnographique pour la cueillette de données a permis au chercheur de faciliter l'interaction avec les participants dans leur vie quotidienne, de participer à leurs activités journalières, comme un membre du groupe social et de prendre en considération le système social et culturel dont ils font partie. L'approche ethnographique menée à l'école musulmane lui a ainsi permis de ne pas dissocier les jeunes participants et leur contexte migratoire du système socioculturel de leur pays de résidence.

Comme cela a été mentionné ci-haut, plusieurs outils de cueillette de données ont appuyé cette méthode de recherche et lui ont donné sa saveur ethnographique.

a. Les notes de terrain : Cet outil de description des évènements, des lieux visités et des comportements a permis au chercheur de se rappeler le plus fidèlement possible des circonstances lors de l'analyse des données. Ce dernier a veillé à prendre des notes en salle de classe, dans la cour de l'école, durant les prières, pendant l'arrivée et la sortie des élèves. Notées sur un cahier, ces informations ont été ensuite retranscrites et classifiées séparément pour chaque participant. À cet effet, deux types de notes ont été préconisés, à savoir les notes substantielles ${ }^{24}$, c'est-à-dire la prise en notes de ce qui est vu et de ce qui est entendu, ainsi que les notes analytiques ${ }^{25}$, qui correspondent aux premières analyses. Le chercheur a ainsi indiqué chaque note substantive avec sa note analytique correspondante. La sélection et les interprétations de ces notes constituaient les premières conversations du chercheur avec ses données.

24 John Brewer, Ethnography, Buckingham, Open University Press, 2000, p. 88. 25 Ibid. 
b. Les entretiens semi-dirigés : Ils constituaient une des étapes les plus importantes parmi tous les modes de collecte de données. Le chercheur a voulu entre autres explorer tous les thèmes qui concernent directement sa recherche, en particulier la construction de l'identité, le rôle de l'école, la perception de l'Islam, le rapport avec la culture majoritaire. Le guide d'entretien élaboré lui a servi de base, mais il a préféré ne pas suivre strictement l'ordre des questions et laisser les participants mener la discussion. Les questions posées étaient directement liées aux réponses pour faciliter l'enchaînement de la discussion. Les informations sont obtenues de manières naturelles, mais sur une période de temps plus longue. Conscient de l'enjeu de l'influence mutuelle entre le chercheur et les sujets, le chercheur n'a pas hésité à donner son avis sur certains sujets au milieu de la discussion quand les participants l'interpellaient directement. Ceci a été le cas, par exemple, quand certains élèves sollicitaient son opinion sur un comportement émis par une surveillante : "et vous? Qu'en pensez-vous? ${ }^{26}$ ". Natalie Schilling-Estes note d'ailleurs les mêmes tendances chez des participants lors de son enquête de terrain qui, une fois à l'aise avec l'enquêteur, lui posent des questions sur l'école, l'intérêt personnel, etc. ${ }^{27}$. Loin d'y voir un biais de la recherche, nous y décelons une manifestation de l'intersubjectivité et de la " conversation » ayant lieu entre le chercheur et les sujets de sa recherche.

c. L'observation ethnographique : Cet outil a permis au chercheur de voir et de faire voir les comportements et les interactions des participants. Sans entrer dans un débat de fond sur les différentes typologies de l'observation, nous croyons comme Christophe Broqua, que l'observation ethnographique adoptée permet de rendre compte à la fois de degrés d'implication variables selon les situations et de différents modes d'obser-

26 Souhaiel Essid, op. cit., p. 73.

27 Natalie Schilling-Estes, "Sociolinguistic Fieldwork ", dans Robert Bayley et Ceil Lucas (dir.), Sociolinguistic Variation: Theory, Methods and Application, Cambridge, Cambridge University Press, 2007, p. 181. 
vation (y compris les entretiens informels avec les participants et le recours à des matériaux divers), tout en favorisant le point de vue de l'acteur afin de retracer comment l'action sociale est formée ${ }^{28}$. Le chercheur a ainsi participé pleinement à la vie quotidienne des participants. Il a partagé avec eux leurs dîners, les prières quotidiennes, fait l'ablution ${ }^{29}$ avec les garçons, joué avec eux au soccer et pris part à des jeux de rôle. Il était souvent impliqué dans leurs conflits et leurs tensions.

d. Le groupe de discussion focalisé : Cette technique a principalement été employée pour identifier les dimensions des notions peu étudiées. Elle a permis au chercheur d'approfondir des concepts comme l'identité musulmane, l'école musulmane, l'islam au Canada, Islam dominé, etc. En plus, ces discussions ont permis d'approfondir les principales questions de recherche d'une façon explicite, à savoir la perception des participants de leur propre identité, de leur religiosité et du rôle de leur école dans cette perception. Cette technique qui repose sur la dynamique de groupe, sans chercher à faire valoir un point de vue, a donné aux participants l'occasion d'exprimer leurs représentations de la réalité sociale et d'euxmême et a permis les interactions entre participants, en favorisant la confrontation de leurs opinions et de leurs expériences personnelles. En outre, cet outil de collecte de données représente une autre manifestation de l'intersubjectivité de plusieurs participants. Adoptant une posture plutôt étique, le chercheur a eu l'opportunité d'observer la « conversation " et les influences mutuelles entre les sujets. Le groupe de discussion focalisé lui a ainsi permis d'accéder à une grande richesse narrative en un temps relativement restreint, tout en laissant la possibilité de garder une position de recul nécessaire à tout effort d'objectivation.

$\overline{28}$ Christophe Broqua, "Observation ethnographique », dans Olivier Fillieuse, Lilian Mathieu et Cécile Péchu, Dictionnaire des mouvements sociaux, Paris, Presses de Sciences Po (P.F.N.S.P.), 2009, p. 379-386.

29 Action de se laver des parties du corps à des fins de purification religieuse. 
e. Le journal de bord : Il fut l'un des meilleurs outils pour assurer la réflexivité du chercheur au cours de la collecte de ses données. Cet outil l'a aidé à mener une réflexion profonde sur son a priori et de retracer le contexte psychologique au moment de la collecte de données. À la suite de chacune des entrevues et des discussions de groupe, le chercheur a consigné dans un journal de bord des informations portant sur le déroulement des rencontres, les sentiments exprimés par les élèves et la dynamique des rencontres elles-mêmes, les sentiments exprimés et la dynamique des échanges. De plus, le journal de bord a servi à la prise en notes de ses propres réflexions et questionnements. En ce sens, il l'a aidé à mener une réflexion profonde sur ses biais essentialistes, sur son statut à l'école et sur son rapport avec l'objet de recherche : chercheur et membre actif de la communauté, foi et rationalisme, immigrant assimilé et immigrant intégré, etc. Bref, le journal de bord lui a permis de mener une réflexion profonde sur lui-même et sur ses a priori. Nous pouvons alors avouer que lors de la dynamique immersion/distanciation, le chercheur s'est lui-même découvert.

Tous ces outils ethnographiques lui ont permis en premier lieu de se distancier de son parcours biographique et relationnel pour pouvoir déchiffrer et analyser les parcours identitaires et les perceptions des participants en dehors des paradigmes partagés. En même temps, ils ont permis de " converser " avec les données dans un processus dynamique bien réfléchi de rapprochement/ distanciation.

\section{Statut du chercheur et terrain de recherche}

La dimension pragmatique de la réalité et des aléas de la recherche et de ses terrains a été traitée sous l'angle du rapport de l'engagement émotif du chercheur en conflit avec sa nécessité de prendre un certain recul et de rester à distance, en quelque sorte, des acteurs qui lui permettent de mieux comprendre et cerner l'objet de recherche. C'est ainsi que nous présentons dans cette section la posture du chercheur et de son terrain qui lui a semblé familier au départ. 


\subsection{Le terrain d'enquête}

Le terrain en question est une école primaire, fondée à la fin des années 1990 par un groupe de musulmans qui se considère soucieux de préserver sa religion minoritaire au sein d'une société occidentale. Les fondateurs souhaitaient créer une "institution qui fonctionne selon les valeurs islamiques ${ }^{30}$ ", selon un parent fondateur dans une discussion informelle, mais qui n'est pas détachée ou isolée de la société, ajoute-t-il. L'école comportait au moment de la recherche, en 2014, 250 élèves et livrait officiellement le programme d'enseignement du Québec. Elle reçoit un financement partiel de la part du gouvernement provincial, conformément à la loi 78 de l'enseignement privé du Québec. En plus du curriculum du ministère de l'Éducation du Québec, l'école livre des programmes particuliers de langue arabe, de morale et de religion musulmane. L'école est officiellement ouverte à tous les enfants, mais uniquement les enfants dont les parents adhèrent à la confession musulmane sont inscrits, quelle que soit leur origine ethnique ou culturelle. À l'image de la religion musulmane, cette école est multiculturelle, multiraciale et multilingue. Elle contient d'excellentes installations sportives et éducatives, entre autres un lieu de prière et des locaux aménagés pour faire l'ablution.

Dans cette recherche, des élèves de Ge année (18 participants) ont été privilégiés. Deux raisons expliquent ce choix. D'abord, c'est le niveau scolaire le plus élevé à l'école (il s'agit d'une école primaire). En plus, l'âge des participants (entre 10 et 12 ans) semble assez convenable pour que les aspects identitaires apparaissent clairement à travers les gestes et les paroles. Dans ses études sur la socialisation politique, Mary Hepburn considère que cet âge constitue une étape marquante de la trajectoire de vie et dans la construction de l'identité des jeunes. Ils sont généralement plus sensibles aux conflits socioculturels et sont souvent appelés à effectuer des choix important ${ }^{31}$.

\footnotetext{
$30 \quad$ Souhaiel Essid, op. cit., p. 77.

31 Mary Hepburn, "Revitalizing Political Socialization Research: An Introduction to the Symposium ", Perspectives on Political Science, vol. 24,
} 
Le terrain de cette recherche est donc, comme toute institution scolaire, un terrain propice aux manifestations identitaires, à partir duquel les acteurs peuvent construire des réseaux qui représentent éventuellement des références à la construction de leur identité. Les élèves passent une grande partie de leurs journées au sein de l'école et de nombreuses activités s'y déroulent. Sur ce terrain de réalisations personnelles, certaines expériences vécues à l'école - scolaire, sportive, artistique, religieuse, culturelle - peuvent être déterminantes dans leurs vies et des relations significatives, soit avec les pairs, soit avec les adultes, peuvent se développer. Partageant implicitement les mêmes valeurs religieuses et culturelles avec ces participants, le chercheur s'est trouvé, en quelque sorte, en terrain connu et n'a point été étonné par les rituels religieux, les prières, la diversité ethnique, le rapport entre les genres, etc., alors que des chercheurs étrangers à la communauté musulmane l'auraient certainement été.

\subsection{Posture du chercheur entre émique et éthique}

Plusieurs recherches abordent le débat sur l'importance et le rôle du chercheur en tant qu'insider/outsider sur le terrain d'enquête. Hammersley et Atkinson évoquent les " mythes " attribués aux rôles d'insider et outsider en ethnographie. Le premier mythe veut qu'un chercheur outsider soit considéré comme le seul capable de mener des recherches valides sur un groupe donné, car il possède l'objectivité nécessaire et la distance émotionnelle. Le deuxième mythe estime que seul le chercheur insider soit capable d'apprécier le véritable caractère de la vie du groupe ${ }^{32}$.

Ainsi, un chercheur étranger au terrain et aux habitudes culturelles et linguistiques dominantes est considéré outsider. Par contre, celui qui semble connaître son terrain avant de s'y insérer est considéré un chercheur insider. Le défi du chercheur insider est de parvenir à construire une relation affective et positive avec les participants et de poser un regard objectif et distancié de son terrain de recherche, tandis que l'obstacle principal pour le 
chercheur outsider réside dans sa capacité à dégager les traits pertinents du groupe ciblé. Il s'agit donc de pouvoir construire le point de vue éthique pour le premier, émique pour le second, selon la terminologie anthropologique de Jean-Pierre Olivier de $\operatorname{Sardan}^{33}$. Évoquant ses expériences en tant qu'enquêteur blanc dans un ghetto noir près de New York, François Bonnet relève l'importance de la maîtrise des codes, des conventions et des coutumes des enquêtés pour des chercheurs outsiders qui seront capables de neutraliser l'étrangeté et de mobiliser d'autres identités ${ }^{34}$. Quant au rôle des insiders, François Bonnet note l'importance de faire partie d'un groupe ethnique ou racial pour réaliser un travail de terrain. Les chercheurs insiders sont « mieux placés pour gagner plus facilement la confiance de leurs interlocuteurs, comprendre les subtilités du langage et les enjeux les plus problématiques des communautés auxquelles ils appartiennent ${ }^{35}$ ". Dans le même sens, Ibrahim Awad adopte la typologie de Rhoda MacRae ${ }^{36}$ et met l'accent sur le chercheur insider in/out et outsider inlout. Ainsi, un chercheur outsider in est culturellement étranger au groupe étudié et reprend sa position externe une fois la collecte des données terminée. Le chercheur outsider out n'a pas d'implication directe dans le groupe étudié. Le chercheur insider in, avec sa proximité sociale, culturelle et linguistique et sa familiarité avec l' "étudié ", a moins de barrières dans son processus de recherche et est capable d'accéder aux données informelles et confidentielles d'une façon approfondie. Le chercheur insider in fait la recherche avec sa communauté et sur lui-même, ce qu'Ibrahim Awad appelle "I-thou research " ${ }^{37}$.

33 Jean-Pierre Olivier de Sardan, "Observation et description en socio-anthropologie ", dans Giorgio Blundo et Jean-Pierre Olivier de Sardan (dir), Pratiques de la description, Paris, Éditions de l'EHESS, vol. 3, 2003, p. 13-40.

34 François Bonnet, "La distance sociale dans le travail de terrain : compétence stratégique et compétence culturelle dans l'interaction d'enquête ", Genèses, $\mathrm{n}^{\circ}$ 4, 2008, p. 57-74.

35 Ibid., p. 60

36 Rhoda MacRae, "Insider" and "Outsider" Issues in Youth Research ", dans Paul Hodkinson et Wolfgang Deicke (dir.), Youth Cultures: Scenes, Subcultures and Tribes, New York, Routledge, 2007, p. 51-62.

37 Ibrahim Awad, "Research as an Act of Love: Ethics, Émigrés, and the Praxis of Becoming Human ", Diaspora, Indigenous, and Minority Education, vol. 8, 
Dans notre cas, le chercheur s'est d'abord considéré comme insider in du fait qu'il partage la même religion que celle des participants et qu'il appartient à la même communauté immigrante. Détecter les marqueurs religieux qui sont si naturels et intuitifs à ses yeux demandait alors un effort de distanciation de sa part afin de comprendre comment ses sujets interprètent ces marqueurs et leur donnent un sens. Croyant ainsi être familier avec ses sujets et son contexte, il s'est muni d'une grille d'observation afin de minimiser l'impact de son a priori.

Toutefois, dès les premiers moments de la recherche empirique et ses premiers contacts avec son terrain, ce statut ou ce privilège a été remis en question. Tout d'abord, puisqu'il se présente souvent comme membre engagé de la communauté et que son objectif consiste à faire valoir le rôle des écoles musulmanes dans le champ du savoir scientifique canadien, il a cru qu'il serait facile pour lui d'accéder aisément à une école musulmane pour effectuer sa recherche. Il a ainsi contacté cinq écoles dans deux villes différentes pour demander une autorisation de séjour pour recherche doctorale. Mais deux écoles ont catégoriquement refusé sa demande sans donner d'explication, deux autres ont d'abord été motivées par la recherche, mais, après des mois d'attente, leurs conseils d'administration ont refusé son projet. Une seule école a répondu positivement à sa requête d'effectuer une recherche auprès de ses élèves. L'école qui a accepté sa demande est située à Montréal. En contactant son directeur avec qui il entretient des rapports étroits, ce dernier a demandé une description du projet de recherche ainsi qu'un descriptif des actions à entreprendre avec les participants durant la période de recherche. Une réponse positive a enfin été reçue durant le mois de décembre 2011.

Une fois sur le terrain, il s'est trouvé au milieu d'un monde de jeunes écoliers québécois bien différents de lui pour ce qui est de la génération, de la culture nationale et aussi de la culture scolaire, étant lui-même éduqué dans une école sécularisée caractérisée par l'absence de marqueurs religieux comme les prières ou le vocable religieux. En effet, après la première rencontre avec les

$n^{\circ}$ 1, 2014, p. 7-20. 
participants, il a fait face à une certaine réticence de la part de plusieurs élèves à son égard : un contact assez formel et le refus de plusieurs élèves de lui adresser la parole. Cette attitude peut s'expliquer par l'ambigüité qui entourait sa présence à l'école à laquelle les élèves ne sont pas habitués, ainsi que par leur attitude habituellement réservée à l'égard des adultes. Une semaine après, seulement 4 élèves sur 19 ont accepté de faire partie de la recherche. Pour renforcer le rapprochement, il a planifié une deuxième rencontre avec les élèves dans une atmosphère plus conviviale et moins formelle, où ils ont pu poser plus de questions et ont manifesté plus d'intérêt. Peu de temps après, tous les formulaires d'acceptation ont été remis et tous ont accepté de participer, à l'exception d'une fille qui dès le début refusait catégoriquement de participer. Mais, après une semaine et face à son isolement, elle a décidé de joindre le groupe de recherche durant les activités de groupes seulement.

Ainsi tout au long de la cueillette de données, le chercheur a dû assumer plusieurs rôles et s'est continuellement vu changer de peau, dépendamment du contexte, tantôt pour se rapprocher de ses sujets (peau du musulman, de personne drôle, empathique) tantôt pour se distancier ou se donner du recul (peau du chercheur, de l'éducateur, de l'adulte). Par ailleurs, la meilleure stratégie qu'il s'est découverte a été d'être "invisible ». D'un côté, ses observations ont principalement porté sur les interactions spontanées entre les participants et sur la vision des participants-élèves de leurs constructions identitaires, particulièrement religieuses, en salle de classe, à la cafétéria où les participants se réunissaient, au gymnase, dans la cour externe pendant les pauses et les heures de dîner, à la salle de prière où les élèves se rendaient chaque jour après le dîner. Le chercheur avait certes plus de temps en salle de classe, mais les moments les plus fructueux de l'observation étaient dans les couloirs de l'école, dans le gymnase, dans la cour extérieure de l'école et durant les prêches de vendredi. La spontanéité des participants et son invisibilité dans ces lieux ont été déterminantes. D'un autre côté, durant les discussions de groupes, c'est la dynamique de groupe qui a souvent conduit les échanges. 
C'est ainsi qu'il a observé que certains participants comme Iheb ${ }^{38}$ et Zaki étaient souvent réticents à s'exprimer; d'un autre côté, Rami et Sami avaient tendance à imposer leur point de vue à tous les garçons, leur autorité était tellement imposante que la plupart des échanges individuels et de groupes étaient manipulés d'avance et les réponses souvent dictées par eux. Certains participants (Imran, Zaki et Rachid) se ralliaient constamment à la majorité. Enfin, bien que le moment du déroulement de la recherche, proche de la date des examens ministériels de la province de Québec, ait constitué une contrainte de temps, il a été en quelque sorte favorable, car il lui a permis de distraire les élèves de sa présence.

Par ailleurs, conscient de certaines thématiques embarrassantes dans la communauté musulmane, le chercheur s'est contenté d'aborder des sujets comme la sexualité, le rapport fille/garçon, la représentation des non-musulmans, etc. d'une façon subtile. Un chercheur étranger à la religion musulmane aurait posé des questions plus directes sur chaque élément qui aurait probablement éveillé sa curiosité. Afin de pouvoir comprendre les multiples déterminations qui peuvent contribuer à la fabrication des relations sociales, il a été contraint d'apprendre ce que Michel Lallement appelle le " désengagement émotionnel ${ }^{39}$ " et à savoir conserver de la distance avec les processus sociaux observés à l'école. Il a ainsi décidé de travailler à prendre conscience de sa propre subjectivité, à mettre au jour les dépendances dont il est luimême prisonnier et à prendre conscience de ses prénotions, de ses valeurs et de la position sociale qu'il occupe. Ce travail ou ce processus lui a alors permis de développer une vigilance ethnographique accrue.

38 Pour des raisons déontologiques, les noms des participants ont été modifiés.

39 Michel Lallement, "Réflexivité, sociologie ", Universalis éducation, Encyclopedia Universalis, http://www.universalis-edu.com/encyclopedie/ reflexivite-sociologie/, site consulté le 11 octobre 2016. 


\section{Intersubjectivité et réflexivité}

Les constats et les résultats de l'étude en question n'auraient pas été possibles sans les diverses et riches interactions et conversations avec les sujets et les données de la recherche ${ }^{40}$, mais aussi sans la réflexivité du chercheur tout au long du processus. En effet, un questionnement d'ordre épistémologique était nécessaire afin de théoriser l'expérience de recherche.

\subsection{Le rapport d'interprétation et d'intersubjectivité}

Comme Jean Piaget qui reconnaît la nécessaire subjectivité de toute connaissance ${ }^{41}$, François Laplantine affirme que « nous ne sommes jamais des témoins objectifs observant des objets, mais des sujets observant d'autres sujets ${ }^{42} \%$. La littérature est un artefact culturel qui reflète les valeurs, les croyances, les conditions sociales, politiques, etc. de l'auteur plus qu'elle ne révèle la réalité. Selon lui, toute écriture n'est qu'une autobiographie du chercheur. Choisir l'épistémologie constructiviste, c'est assumer la subjectivité des connaissances et ainsi de tout savoir et de toute recherche. Ainsi, à chaque étape de la recherche (choix théoriques et méthodologiques, observations, prise de notes de recherche, conduite des entrevues, analyse des données, etc.), c'est la subjectivité, l'intégrité personnelle et la sensibilité du chercheur qui sont mises en jeu. Il s'agit donc de prendre conscience de cette subjectivité caractéristique de la recherche et de l'assumer, tout en assurant certains critères de rigueur et en en reconnaissant les limites.

Il va sans dire que cette subjectivité fait la richesse des études qualitatives tant au niveau de la collecte que de l'interprétation des données recueillies et reconstruites ${ }^{43}$. En effet, nombre

$\overline{40}$ Jean-Marie Van der Maren, « La recherche qualitative, instrument stratégique

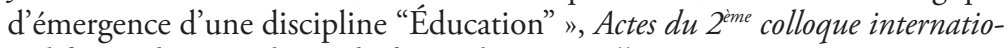
nal francophone sur les méthodes qualitatives, Lille, juin 2009.

41 Jean Piaget, Biologie et connaissance, Paris, Gallimard, 1967.

42 François Laplantine, L'anthropologie. Paris, Payot, 1995, p. 168.

43 Marta Anadón et Lorraine Savoie-Zajc, "L'analyse qualitative des données ", Recherches qualitatives, vol. 28, no 2, 2009, p. 1-7 ; Jane Mills, Ann Bonner et Francis Karen, "The Development of Constructivist Grounded Theory ", 
d'auteurs s'accordent à dire que la théorisation ne peut avoir lieu sans l'implication personnelle du chercheur (expériences personnelles, connaissances théoriques, connaissances professionnelles, capacité réflexive, curiosité, ouverture, flexibilité, discipline, écoute, patience, etc. $)^{44}$. Pour Kathy Charmaz, c'est l'interaction entre le chercheur et les participants qui donne naissance aux données ${ }^{45}$. En anthropologie, la rencontre de deux subjectivités permet la création de situations " nouvelles » appelées l'intersubjectivité $^{46}$. Dans un processus où chercheur et sujet s'observent et s'influencent mutuellement, tous les deux deviennent coproducteurs de la connaissance. Dès lors, il importe que la situation d'interaction sociale soit la plus consciente possible et que le chercheur fasse preuve de réflexivité ${ }^{47}$. La subjectivité est ainsi loin de constituer une entrave à la recherche. En l'assumant et en restant réflexifs, nous sommes capables de la transformer en un atout. Dans notre cas, sachant qu'il ne pouvait se soustraire à cette subjectivité, le chercheur l'a assumée. Comme il ne peut être culturellement neutre, il est parti avec son bagage religieux et avec son expérience personnelle et professionnelle comme ex-enseignant titulaire à l'école musulmane.

International Journal of Qualitative Methods, vol. 5, n 1, 2006, http://www. ualberta.ca/_iiqm/backissues/5_1/html/mills.htm, site consulté le 12 mars 2011. Francine Gratton, "La théorisation ancrée pour proposer une explication du suicide des jeunes ", dans Henri Dorvil et Robert Ayer (dir.), Problèmes sociaux, Tome 1 : Théories et méthodologies, Sainte-Foy (QC), Presses de l'Université du Québec, 2001, p. 306-334 ; Lorraine Savoie-Zajc, " La recherche qualitative/interprétative en éducation ", dans Thierry Karsenti et Lorraine Savoie-Zajc (dir), Introduction à la recherche en éducation, Sherbrooke, Éditions du CRP, 2000, p. 171-198 ; Jean-Marie Van der Maren, op. cit. ; David Thomas, "A General Inductive Approach for Analyzing Qualitative Evaluation Data ", American Journal of Evaluation, vol. 27, $\mathrm{n}^{\circ} 2$ 2, 2006, p. 237-246.

Kathy Charmaz, Constructing Grounded Theory: A Practical Guide through Qualitative Analysis, Londres, Thousand Oaks, New Delhi, SAGE, 2006.

46 François Laplantine, L'anthropologie, Paris, Payot, 1995.

Bertrand Troadec, "Approches culturelle et interculturelle comparative. Vers une intégration de paradigmes complémentaires ", Actes du VIII'me Congrès de l'Association pour la Recherche Interculturelle (ARIC), Université de Genève, septembre 2001. 
Il a en effet longuement pensé à son objet de recherche, ses motivations, ses objectifs et ses attentes. Par la suite, au contact des participants, il a appris leurs propres façons de voir, d'analyser et d'interpréter. En effet, les sujets de la recherche, par leur culture générationnelle, leur éducation et leurs expériences, entretiennent des rapports différents à la culture du chercheur. Nous pouvons alors affirmer que le processus de recherche et d'objectivation des données ne peut être dissocié des processus d'intersubjectivité. De l'interaction entre chercheur et sujets et surtout de cette prise de conscience de la part du chercheur des différences, des similitudes et des influences mutuelles, est née l'intersubjectivité de la recherche. Dès lors, il importe que la situation d'interaction sociale soit la plus consciente possible et que le chercheur fasse preuve de réflexivité ${ }^{48}$.

\subsection{Réflexivité et vigilance ethnographique}

Pour modérer sa subjectivité, le chercheur a adopté ce que Joëlle Morrissette, Didier Demazière et Matthias Pepin appellent " vigilance ethnographique ${ }^{49}$ ", tout en restant ouvert à de nouvelles découvertes.

La vigilance ethnographique constitue à la fois un concept théorique pour traduire le processus réflexif qui conduit le chercheur à prendre des décisions affectant le cours de sa démarche et un levier pour objectiver sa pratique d'enquête. Ainsi, la démarche ethnographique n'est pas un simple modèle à mettre en œuvre ou à un ensemble de normes codifiées à appliquer, elle exige du chercheur de s'imprégner du terrain, de ses aspérités et de sa singularitée ${ }^{50}$. Ainsi, la démarche de recherche se construit au fil des interactions dans lesquelles le chercheur est engagé sur

\footnotetext{
$48 \quad$ Ibid.

49 Joëlle Morrissette, Didier Demazière et Matthias Pepin, «Vigilance ethnographique et réflexivité méthodologique ", Recherches qualitatives, vol. 33, $\mathrm{n}^{\circ}$ 1, 2014, p. 9-18.

50 Charles Soukup, "The Postmodern Ethnographic Flaneur and the Study of Hyper-Mediated Everyday Life ", Journal of Contemporary Ethnography, vol.42, no 2, 2012, p. 226-254.
} 
son terrain ${ }^{51}$. De ce fait, la démarche s'adapte et se négocie au fil de l'expérience du chercheur et les questionnements sont toujours renouvelés. Dans sa dimension théorique, la vigilance ethnographique tient lieu de porte d'entrée qui permet de s'interroger aussi bien sur l'objet de l'enquête que sur les concepts qui balisent sa pratique. La position du chercheur n'est donc pas fixée une fois pour toutes, mais varie en fonction des contextes, des moments et des personnes avec lesquels il interagit sur son terrain.

Dans le cas de la recherche sur la construction de l'identité des jeunes dans une école ethno-religieuse, la distanciation de l'objet de recherche est certes un défi dans une recherche de type constructiviste interprétativiste où un rapport étroit existe avec l'objet et les sujets de la recherche. Ce défi est encore plus grand vu la difficulté d'entretenir des rapports purement impersonnels ou professionnels avec les sujets. Comme en Islam, l'absence de médiation entre la divinité et les humains écarte tout rapport impersonnel de l'individu avec les autres. Les interprétations dépendent ainsi, en partie, des représentations et des schèmes propres au chercheur. Ce dernier risque alors la distorsion et la projection ethnocentrées ${ }^{52}$, surtout qu'il prend comme contexte d'étude sa propre religion, acquise depuis la naissance et largement défendue. En effet, lorsque notre propre identité est étudiée, nous ne pouvons pas prétendre à la neutralité, car nous sommes imprégnés de valeurs culturelles et de convictions religieuses dont il est difficile, voire impossible de se détacher, mais le chercheur doit travailler à prendre conscience de cette projection.

Durant la collecte des données, le chercheur était aussi conscient que sa présence sur le terrain influence les interactions entre les participants. Ceci a été modéré par sa volonté de laisser

\footnotetext{
51 Didier Demazière, François Horn et Marc Zune, «La socialisation dans les "communautés" de développement de logiciels libres ", Sociologie et sociétés, vol. 41, n' 1, 2011, p. 217-238.

52 Bertrand Troadec, Approche culturelle et interculturelle comparative : Vers une intégration de paradigmes complémentaires, Communication présentée au VIIle Congrès de l'Association pour la Recherche InterCulturelle (ARIC), Recherche et pratique interculturelles : nouveaux espaces, nouvelles complexités?, Genève, Suisse, septembre 2001.
} 
les participants s'exprimer librement sans trop intervenir pour orienter ou encadrer les discussions. Ce choix a parfois coûté cher à son image comme éducateur, puisqu'il a dû laisser passer certains actes d'intimidation entre certains participants pour ne pas biaiser les discussions. Il a aussi veillé à rester fidèle aux propos notés et à éviter les écueils de l'extrapolation en prévoyant des questions de recoupement et en revenant le plus possible à la terminologie utilisée par les participants. Durant l'analyse des données, avant de coller aux participants des a priori, il a essayé de comprendre leurs propos et leurs réactions dans leur contexte (histoire de vie, problèmes de famille, parcours scolaires, etc.), et ce, indépendamment de toute influence culturelle et religieuse.

C'est ainsi qu'afin d'assurer la rigueur et la fiabilité des données recueillies, le chercheur s'est préoccupé, pendant et après le processus de collecte et d'analyse des données, de préciser ses motivations personnelles, d'établir sa propre autocritique, de retracer sa démarche, mais aussi d'assurer la triangulation des résultats en diversifiant les sources d'information.

L'analyse de sa réflexivité lui a ainsi permis d'appliquer les outils de l'analyse à son propre travail et donc de faire partie de son sujet d'étude. À travers cette démarche méthodologique, le chercheur a découvert d'autres voies par où et comment enquêter : les discussions en dyade, les rencontres informelles avec les parents, les diners-partage avec les filles, les matchs de soccer avec les garçons, etc. Afin de conserver une démarche rigoureuse et rationnelle, il était nécessaire pour lui de travailler à construire une compréhension du sens donné par chaque participant dans le processus d'échange et de ne pas dénier sa propre expérience du monde, faisant ainsi partie de son objet de recherche. Un autre défi de la recherche est sans aucun doute de s'affronter soi-même pour pousser l'explication des phénomènes inconnus dans le milieu éducatif comme les écoles musulmanes, l'identité des jeunes musulmans au Canada, l'islam en Occident, etc. 


\section{Statut du chercheur et résultats de recherche}

Les résultats de cette étude ont démontré que les jeunes participants ont une relation privilégiée avec l'Islam, au moins par le milieu familial et scolaire, mais nombreux trouvent que l'expérience de l'ordre du "sacré » a une forte charge émotionnelle. Dans cette dernière section, nous présentons les résultats de l'étude qui se manifestent à travers les conceptions que construisent les jeunes participants de leur religiosité, ensuite nous démontrons comment le processus conversationnel entre le chercheur, ses a priori et les données recueillies a contribué à sa distanciation et à son positionnement réflexif.

\subsection{Les résultats de l'étude}

Trois conceptions de l'Islam et de l'islamité se sont construites à partir des marqueurs que les jeunes développent et de la religiosité qu'ils expriment : la conception d'un Islam familial reçu autour duquel se greffent deux autres manières de vivre cette religion : un Islam recomposé par des jeunes qui cherchent à donner du sens à leur vie et un Islam universel qui dépasse les frontières géographiques et ethniques.

\section{a. L'Islam familial hérité}

Les récits de vie des jeunes participants démontrent que la culture parentale et scolaire façonne les modes de représentation de soimême dans un environnement social perçu comme hostile. Face à la pratique de l'arabe dialectal de plus en plus fragilisé et des valeurs culturelles orientales menacées, la valorisation de la religion permet à certains parents de préserver d'autres biens de l'histoire familiale qui a porté le projet d'immigration. À titre d'exemple, l'expérience religieuse de Khamel est étroitement liée à l'histoire de guerre en Palestine. Sa religiosité est intimement liée à la terre natale de ses parents, il évoque souvent leurs biens confisqués et, durant ses activités religieuses, les références au pays d'origine sont remarquablement distinctives : drapeau palestinien, habits traditionnels et folklore populaire. 
C'est par la religion que se fait encore l'essentiel de la transmission culturelle et des valeurs sociales et morales. Construite autour d'une reproduction quasi fidèle de la religion parentale, la religiosité de ces participants est une filiation. Ils reçoivent un Islam populaire qui est mêlé aux traditions du pays d'origine. Cependant, cet héritage religieux peut produire une attitude extrême pour devenir une forme de défense et de protection et une forme de refus pour exprimer son rôle de compensation et de remède aux frustrations. Certains participants affichent leur qualité de musulman avec une teinte culturelle assez marquée : Palestinien/musulman, mais également, une négation culturelle assez marquée : musulman/non-Algérien. Face à la culture majoritaire, ces jeunes revendiquent leur appartenance à leurs cultures d'origine et à leur religion héritée d'une façon ostentatoire.

b. L'Islam reconstruit

L'Islam de la majorité des jeunes participants est travaillé par l'expérience familiale et scolaire et par le contexte diasporique, à savoir la culture occidentale. Ainsi, ces participants insistent sur une spiritualité qui se démarque des modes occidentales et qui s'oppose à l'image d'un Islam de pure obéissance, transmis par la tradition et devenu de plus en plus décontextualisée, selon les jeunes.

Rihab, par exemple, n'hésite pas à accuser une surveillante quand elle lui a interdit de regarder son magazine préféré avec ses amies : "Elle a dit c'est haram. Elle ment». Julie réplique, dans un autre contexte, à d'autres surveillantes qui cherchent toujours à séparer les filles des garçons quand ils s'entretiennent ensemble devant la salle de classe ou dans les couloirs de l'école : "Je sais qu'elles mentent. L'autre jour, l'imam de la mosquée a dit que c'est pas haram si les filles et les garçons jouent ou étudient ensemble". Les participants utilisent souvent les expressions "archaïques" et " un truc de vieux" pour qualifier certaines exigences religieuses.

L'Islam reconstruit par les jeunes est, ainsi, l'expression d'une foi teintée d'une expérience personnelle. Le jeûne du ramadan, 
par exemple pour Rami, Emna et Marwa, reprend son sens spirituel avec la multiplication des séances de prières et d'invocation spirituelle et l'implication dans des activités bénévoles dans les centres islamiques de leurs régions. Ainsi, ces participants dénoncent le ramadan comme une simple exécution conforme à la tradition ou un parcours commercial sans fin. Il semble ainsi que cette libre réappropriation de l'Islam est surtout le fait de jeunes participantes (10 filles sur 13 participants). Les filles s'appuient sur la religion pour sortir. Manal, Fatma et Samira disent qu'elles ratent rarement une rencontre ou une activité aux mosquées qui leur sont proches, car «c'est une occasion pour sortir et rencontrer les filles ", dit Fatma. L'islamité s'accomplit, donc, dans une dynamique de changement et d'innovations malgré la fidélité proclamée aux valeurs de sources, aux fondements de l'Islam.

\section{c. L'Islam universel}

Dans une situation de migration, les dimensions psychologique et sociologique convoquent le besoin et la nécessité d'une intégrité et d'une continuité, parfois menacée. Pour cette raison, les jeunes paraissent conscients que leurs pratiques religieuses sont partagées par de nombreux musulmans du monde. Ils partagent une solidarité spirituelle (prières et sermons) et physique (jeûne et fêtes) qui les lie symboliquement aux croyants par-delà les frontières géographiques. Être Algérien, Marocain ou Irakien pour les jeunes est une origine culturelle. Au cours d'une discussion entre garçons, un participant déclare : "Pour moi, c'est quand on se met à table je me rappelle que je suis Tunisien ou quand on rentre au pays". Iheb se montre plus ouvert: "J'ai pas de problèmes de vivre ailleurs, mais je serai toujours musulman canadien".

Les jeunes ne nient pas leur canadianité et croient, au contraire, que l'appartenance au Canada leur a ouvert la porte à un Islam universel. En effet, en se pensant "Canadiens musulmans », les appartenances culturelles des jeunes d'origines diverses ne s'opposent plus à ce qui symbolise l'attachement et la fidélité aux valeurs 
familiales ou à l'intériorisation de nouvelles valeurs culturelles au travers d'une unique appartenance géographique. C'est ainsi que l'émergence de représentations d'une appartenance non ethnique et transnationale à travers le recours à la notion de oummah ${ }^{53}$ fournit aux sujets une continuité entre des composantes communes familiales et historiques tout en assumant pleinement leurs vécus dans le pays où ils sont. Dans une discussion de groupe entre garçons autour des perspectives d'avenir de chacun, Imran évoque en toute confiance : "Je suis né à Montréal, je suis Canadien, mais je peux vivre n'importe où il y a des musulmans partout au monde".

Cependant, dans cette quête d'universalité, plusieurs jeunes tendent volontairement ou non vers une forme d'auto-exclusion. La valorisation de l'appartenance religieuse semble exclure certains jeunes participants des autres cercles d'appartenance non religieuse.

\subsection{La démarche conversationnelle}

Croyant d'abord que les conceptions des jeunes de leur islamité constituent une réaction face à un vécu souvent dénoncé et le résultat des appartenances contradictoires, le processus d'objectivation et la démarche conversationnelle ont permis au chercheur de comprendre que les jeunes participants s'affichent différemment. Les trois conceptions religieuses énumérées ci-haut représentent un cheminement en négociation qui se construit par interaction avec les différents groupes d'appartenance.

Se distancier du discours interne dominant qui légitime une identité " religiocentrique " a permis au chercheur de découvrir que la majorité des participants construisent leur identité à partir de l'interaction dynamique avec leur environnement culturel et social. Dans un contexte où les jeunes issus de l'immigration musulmane cherchent à faire leur place dans la société en tant que musulmans, les participants, chacun à sa façon, se montrent particulièrement ardents dans la défense de leur identité musul-

53 Mot arabe qui renvoie au concept de la communauté des musulmans dans le monde quelles que soient leurs origines ou leurs appartenances. 
mane. Cette identité est souvent conçue en termes de binarité, étant donné la bi-culturalité de ces jeunes s'inscrivant à la fois dans la culture de leur pays d'origine où l'Islam est souvent valorisé et sacralisé à travers la famille, l'école et la société, et dans la culture du pays de résidence où l'Islam fait partie de la sphère privée et souvent méconnue. Plusieurs participants s'orientent vers la construction d'une identité qui se caractérise par une distanciation et une remise en question religieuse pour aboutir à une démarche plus personnalisée de leurs choix religieux et identitaires. Deux tendances identitaires se développent ainsi. Iheb, Manal, Fatima, Iheb, Imran, Marwa, Zaki et Samira perçoivent leur identité musulmane en contexte de résistance à la fois contre le discours musulman et le discours de dominance. Quant à Zakia, Bochra, Imen, Rachid, Bochra, Rami, Rihab, Yomna et Julie, ils démontrent une prise de conscience d'une identité musulmane forte, mais elle est redéfinie. Hybride, elle doit composer avec d'autres qui lui sont parfois hostiles. Ils aspirent à une transformation sociale qui garantit leur légitimation.

Cependant, les conceptions officielles semblent peser sur les expériences et les conceptions des participants. Dans ce sens, les jeunes n'évoluent pas vers une indifférenciation de leurs héritages culturel et religieux. La majorité ne semble pas souhaiter se diriger vers une fusion de différentes conceptions et la disparition de certaines dans d'autres. Au contraire, ils revendiquent leur droit d'être eux-mêmes, comme ils l'entendent. Manal, Marwa et Rihab, par exemple, entendent pouvoir être musulmanes à part entière dans la société dans laquelle elles vivent. Néanmoins, le concept oummah reconnaît la " déterritorialisation " de l'identité musulmane et permet de résoudre le paradoxe d'une bi-culturalité souvent génératrice de conflits identitaires. L'identité musulmane des jeunes semble donc multiple, mais ne se fragmente pas, puisqu'elle est articulée à un ensemble d'expériences religieuses et culturelles, passées et présentes, vécues au fil du temps, puis pensées pour produire le sens que nous appelons identité musulmane. 


\section{Conclusion}

La recherche nécessite la prise en considération d'une posture singulière. Elle n'est pas un processus linéaire et rationnel. Ce processus est, au contraire, percé et secoué par de multiples émotions suscitées par des expériences personnelles et des rencontres significatives. Dans l'étude sur la construction identitaire des jeunes musulmans, le chercheur, en s'engageant dans une analyse réflexive sur ses actions, était capable de faire face à des situations non familières et, parfois, problématiques de différentes façons. La réflexion s'est déroulée sur deux niveaux : le premier s'identifie à l'analyse réflexive en cours d'action, durant l'enquête, en modifiant ses actions selon la situation réelle. Puis, le deuxième niveau concerne l'analyse réflexive sur l'action comportant une démarche plus approfondie de nature et plus critique. Ce second niveau de réflexion l'a conduit à prendre une distance vis-à-vis de sa pratique quotidienne et à s'interroger sur le contenu et les raisons de ses démarches et de ses décisions. 


\section{Bibliographie}

Albero, Brigitte, "L'étude de cas : une modalité d'enquête difficile à cerner ", dans Brigitte Albero et Nicole Poteaux (dir.), Enjeux et dilemmes de l'autonomie. Une expérience d'autoformation à l'université : étude de cas, Paris, Maison des Sciences de l'Homme, coll. "praTICs ", 2010, p. $15-25$.

Anadón, Marta et Lorraine Savoie-Zajc, "L'analyse qualitative des données », Recherches qualitatives, vol. 28, $\mathrm{n}^{\circ}$ 2, 2009, p. 1-7.

Awad, Ibrahim, "Research as an Act of Love: Ethics, Émigrés, and the Praxis of Becoming Human ", Diaspora, Indigenous, and Minority Education, vol. 8, $\mathrm{n}^{\circ}$ 1, 2014, p. 7-20.

Barus-Michel, Jacqueline, "Le chercheur, premier objet de la recherche ", Bulletin de psychologie, $\mathrm{n}^{\circ} 377$, tome 39, 1986, p. 801-804.

Bonnet, François, «La distance sociale dans le travail de terrain : compétence stratégique et compétence culturelle dans l'interaction d'enquête ", Genèses, no 4, 2008, p. 57-74.

Bourdieu, Pierre, "The practice of reflexive sociology ", dans Pierre Bourdieu et Löic J. D. Wacquant, An Invitation to Reflexive Sociology, Cambridge, Polity, 1992, p. 217-260.

Brewer, John, Ethnography, Buckingham, Open University Press, 2000.

Broqua, Christophe, "Observation ethnographique ", dans Olivier Fillieuse, Lilian Mathieu et Cécile Péchu, Dictionnaire des mouvements sociaux, Paris, Presses de Sciences Po (P.F.N.S.P.), 2009, p. 379-386.

Charmaz, Kathy, Constructing Grounded Theory: A Practical Guide through Qualitative Analysis, Londres, Thousand Oaks, New Delhi, , SAGE, 2006.

Creswell, John, Qualitative Inquiry and Research Design: Choosing among Five Traditions, Thousand Oaks (CA), SAGE, 1998.

Czarniawska, Barbara, Narrating the Organization: Dramas of Institutional Identity, Chicago, The University of Chicago Press, 1997.

Demazière, Didier, François Horn et Marc Zune, "La socialisation dans les "communautés" de développement de logiciels libres ", Sociologie et sociétés, vol. 41, $\mathrm{n}^{\circ} 1,2011$, p. 217-238.

Essid, Souhaiel, La construction de l'identité dans une école indépendante: Le cas d'une école musulmane à Montréal, thèse de doctorat, Ottawa, Université d'Ottawa, 2015.

Foucart, Jean, "La recherche sociale entre immersion et distanciation ", Pensée plurielle, vol. 2, nos 30-31, 2012, p. 271-282. 
Girod-Séville, Martine et Veronique Perret, « Fondements épistémologiques de la recherche ", Raymond-Alain Thiétart (dir.), Méthodes de recherche en management, Paris, Dunod, 1999, p. 13-33.

Gratton, Francine, "La théorisation ancrée pour proposer une explication du suicide des jeunes ", dans Henri Dorvil et Robert Ayer (dir.), Problèmes sociaux, Tome 1 : Théories et méthodologies, Sainte-Foy (QC), Presses de l'Université du Québec, 2001, p. 306-334.

Hamel, Jacques, Étude de cas et sciences sociales, Paris, L'Harmattan, coll. "Outils de recherche ", 1997.

Hammersley, Martyn et Paul Atkinson, Ethnography: Principles in Practice, $3^{\mathrm{e}}$ edition, Londres, Routledge, 2007.

Hammersley, Martyn et Roger Gomm, «Introduction », dans Roger Gomm, Martyn Hammersley et Peter Foster (dir.), Case Study Method: Key Issues, Key Texts, Londres, SAGE, 2000, p. 1-16.

Hepburn, Mary, «Revitalizing political socialization research: an introduction to the symposium ", Perspectives on Political Science, vol. 24, $\mathrm{n}^{\circ} 1$, 1995, p. 5-6.

Lallement, Michel, "Réflexivité, sociologie ", Universalis éducation, Encyclopædia Universalis, http://www.universalis-edu.com/encyclopedie/reflexivite-sociologie/, site consulté le 11 octobre 2016.

Laplantine, François, L'anthropologie, Paris, Payot, 1995.

Marc, Edmond, "Connaissance de la vérité et vérité de la connaissance ", Société française de Gestalt, vol. 1, n 34, 2008, p. 11-28.

MacRae, Rhoda, "Insider" and "Outsider" Issues in Youth Research", dans Paul Hodkinson et Wolfgang Deicke (dir.), Youth Cultures: Scenes, Subcultures and Tribes, New York, Routledge, 2007, p. 51-62.

Mills, Jane, Ann Bonner et Francis Karen, "The Development of Constructivist Grounded Theory ", International Journal of Qualitative Methods, vol. 5, n 1, 2006, http://www.ualberta.ca/ -iiqm/backissues/5_1/html/mills.htm, site consulté le 12 mars 2011.

Milroy, Lesley, Language and Social Networks, Oxford, Blackwell, 1980.

Morrissette, Joëlle, Didier Demazière et Matthias Pepin, "Vigilance ethnographique et réflexivité méthodologique ", Recherches qualitatives, vol. 33, no 1, 2014, p. 9-18.

Piaget, Jean, Biologie et connaissance, Paris, Gallimard, 1967.

Poisson, Yves, La recherche qualitative en éducation, Québec, Presses de l'Université du Québec, 1991.

Sardan, Jean-Pierre Olivier de, "Observation et description en socioanthropologie », dans Giorgio Blundo et Jean-Pierre Olivier de Sardan 
(dir.), Pratiques de la description, Paris, Éditions de l'EHESS, vol. 3, 2003, p. 13-40.

Savoie-Zajc, Lorraine, "La recherche qualitative/interprétative en éducation ", dans Thierry Karsenti et Lorraine Savoie-Zajc (dir), Introduction à la recherche en éducation, Sherbrooke, Éditions du CRP, 2000, p. 171-198.

Schilling-Estes, Natalie, "Sociolinguistic fieldwork », dans Robert Bayley et Ceil Lucas (dir.), Sociolinguistic Variation: Theory, Methods and Application, Cambridge, Cambridge University Press, 2007, p. 165-189.

Stake, Robert, "Case studies ", dans Norman Denzin et Yvonna Lincoln (dir.), Handbook of Qualitative Research, Thousand Oaks (CA), SAGE, 2000, p. 435-454.

Soukup, Charles, "The Postmodern Ethnographic Flaneur and the Study of Hyper-Mediated Everyday Life ", Journal of Contemporary Ethnography, vol.42, no 2, 2012, p. 226-254.

Thomas, David, «A General Inductive Approach for Analyzing Qualitative Evaluation Data ", American Journal of Evaluation, vol. 27, n 2, 2006, p. 237-246.

Troadec, Bertrand, "Approches culturelle et interculturelle comparative: vers une intégration de paradigmes complémentaires ", Actes du VIII eme Congrès de l'Association pour la Recherche Interculturelle (ARIC), Université de Genève, septembre 2001.

Van der Maren, Jean-Marie, "La recherche qualitative, instrument stratégique d'émergence d'une discipline "Éducation" ", Actes du $2^{\text {eme }}$ colloque international francophone sur les méthodes qualitatives, Lille, juin 2009.

Walters, Sue, " "Case Study" or "Ethnography”? Defining Terms, Making Choices and Defending the Worth of a Case ", dans Geoffrey Walford (dir.), Methodological Developments in Ethnography (Studies in Educational Ethnography), vol. 12, Emerald Group Publishing Limited, 2007, p. 89-108. 